\title{
DE LA INVESTIGACIÓN AL INVESTIGADOR. ADAPTANDO SERVICIOS EN LA BIBLIOTECA RECTOR GABRIEL FERRATÉ
}

\author{
From research to researchers. Adapting services at the \\ Rector Gabriel Ferraté Library
}

\section{Miquel Codina-Vila y Ruth Íñigo}

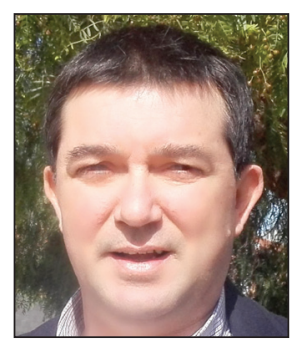

Miquel Codina-Vila es director de la Biblioteca Rector Gabriel Ferraté, de la Universitat Politècnica de Catalunya-Barcelona TECH (UPC), desde 2006. Anteriormente había ejercido como bibliotecario y director de varias bibliotecas en la UPC. Es autor de artículos y comunicaciones sobre gestión de bibliotecas, servicios en bibliotecas universitarias y aplicación de la tecnología en bibliotecas. $\mathrm{Ha}$ colaborado en proyectos internacionales como DART-Europe E-theses portal, Tempus project Maracanda: University libraries towards the new millennium y Tempus Tacis Tramal project: Ulaanbaatar - Barcelona-Dublin.

http://orcid.org/0000-0001-7253-1191

miquel.codina@upc.edu

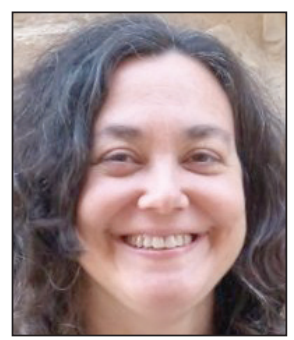

Ruth Íñigo es responsable de la Unidad de Servicios de Apoyo a la Investigación de la Biblioteca Rector Gabriel Ferraté, de la Universitat Politècnica de Catalunya-Barcelona TECH (UPC), desde 1996. Anteriormente había ejercido como bibliotecaria, también en la UPC. Ha publicado trabajos sobre publicación en acceso abierto y depósitos institucionales, propiedad intelectual, y servicios de información especializados. Ha colaborado en proyectos internacionales como Communia: the European network on the digital public domain y Tempus Tacis Tramal Project: Ulaanbaatar-Barcelona-Dublin.

http://orcid.org/0000-0001-7153-6564

ruth.inigo@upc.edu

Universitat Politècnica de Catalunya-Barcelona TECH (UPC), Biblioteca Rector Gabriel Ferraté Jordi Girona, 1-3. 08034 Barcelona, España

\section{Resumen}

Los servicios a la investigación han sido tradicionalmente una de las prioridades de las bibliotecas universitarias, especialmente en aquellas universidades con vocación investigadora. Nos hallamos en un entorno cambiante en el que los investigadores tienen necesidades a menudo temporales. A las bibliotecas les resulta complicado dar respuestas adecuadas ante fenómenos como el acceso abierto a la información, crisis en el modelo de suscripción a las revistas, necesidad de más procesos para evaluar el trabajo de los investigadores, internacionalización, diversidad de perfiles de investigador, etc. Debido a ello, los servicios bibliotecarios amplían el foco de su oferta, dedicando más atención a dar apoyo los investigadores en su rol de autores, y adaptándose a las prioridades de la institución en cada momento. Este artículo presenta las respuestas que la Biblioteca Rector Gabriel Ferraté, de la Universitat Politècnica de Catalunya-BarcelonaTech, está ofreciendo a estos nuevos retos.

\section{Palabras clave}

Bibliotecas universitarias; Servicios a la investigación; Investigadores; Producción científica; Depósitos institucionales; Evaluación de investigadores; Acceso abierto; Biblioteca Rector Gabriel Ferraté; Universidad Politécnica de Cataluña-BarcelonaTech, UPC.

\begin{abstract}
Research services have traditionally been a priority of university libraries, particularly at universities with a research vocation. We are in a changing environment in which researchers often have temporary needs. Libraries find it difficult to provide adequate answers to phenomena such as open access to information, crisis in the journal subscription model, need of more processes to evaluate the work of researchers, internationalization, diversity of researcher profiles, etc.). As a result, library services extend the focus of their offerings, giving more support to researchers in their role as writers, and adapting to the
\end{abstract}


priorities of the institution at all times. This article explores the responses of the Universitat Politècnica de CatalunyaBarcelonaTech's Rector Gabriel Ferraté Library to these new challenges.

\section{Keywords}

University libraries; Research services; Researchers; Scientific output; Institutional repositories; Researcher assessment; Open access; Rector Gabriel Ferraté Library; Polytechnical University of Catalonia-BarcelonaTech, UPC.

Codina-Vila, Miquel; Íñigo, Ruth (2015). "De la investigación al investigador. Adaptando servicios en la Biblioteca Rector Gabriel Ferraté". El profesional de la información, v. 24, n. 5, pp. 648-655.

http://dx.doi.org/10.3145/epi.2015.sep.13

\section{Introducción}

En los últimos años ha habido una progresiva transformación en la forma en que las universidades y los investigadores desempeñan su labor investigadora. Algunos de los factores que han contribuido a ello son:

- La producción científica, expresada en términos de cantidad e impacto, es el elemento más utilizado por agencias de financiación, gobiernos, etc., para valorar los resultados o las posibilidades en un escenario caracterizado por la tensión entre competencia y colaboración (Birnholtz, 2007; Iribarren-Maestro et al., 2015).

- La colaboración entre instituciones a nivel nacional e internacional se ha incrementado en los últimos años ( $F e$ cyt, 2014), fruto de los mecanismos de financiación de la investigación estatal y europea, y gracias a iniciativas institucionales de promoción de la internacionalización de los estudios, y a favor de la movilidad de investigadores y estudiantes.

- El contexto económico, definido por una fuerte crisis, está afectando directamente la labor de las instituciones académicas (INE, 2015). Las universidades intentan aprovechar el impulso de los últimos años pero los objetivos son ahora garantizar la continuidad a los proyectos ya iniciados, descubrir nuevas oportunidades y asegurar la viabilidad de iniciativas emergentes (Michavila, 2013).

- Los canales de la comunicación científica continúan evolucionando, incorporando nuevas funciones TIC en consonancia con los hábitos de una nueva generación de investigadores (Van-Noorden, 2014; Nández; Borrego, 2013).

- Desde un punto de vista competitivo, se trata de poner en valor las propias fortalezas, las de la institución y las de los grupos e investigadores que están detrás de cada proyecto. La investigación Ilama a la investigación. El énfasis se pone en la difusión y visualización de los logros conseguidos y su retorno a la sociedad. Por ello se hace necesaria una mayor y más eficiente proyección de los resultados de la investigación a través de canales propios y externos.

\section{El investigador en el eje de la actividad investigadora}

El Campus Nord de la Universitat Politècnica de Catalunya (Cnupc) es una estructura académica formada por unas 10.000 personas vinculadas a 3 centros docentes -Escola Tècnica Superior d'Enginyeria de Telecomunicació de Barcelona (Etsetb), Escola Tècnica Superior d'Enginyeria de Camins Canals i Ports de Barcelona (Etseccpb) y Facultat
d'Informàtica de Barcelona (FIB)-, 19 departamentos, diversos centros de investigación y servicios científico-técnicos, así como el propio rectorado y algunos servicios centralizados de gestión y administración. La concentración de actividades de investigación lo convierte en el polo estatal más importante en investigación TIC y en ingeniería civil a nivel universitario. En el Cnupc se genera hasta el 36\% del total de la investigación de la Universitat Politècnica de Catalunya (UPC, 2008; 2014b).

\section{La investigación llama a la investigación}

En este entorno, la Biblioteca Rector Gabriel Ferraté (BRGF), como elemento común del Campus Nord, se concibió como un servicio de características capaces de responder a las demandas del mismo. La investigación es uno de los pilares de servicio de la BRGF y ya desde su fundación en 1996 contó con una Unidad de Servicios a la Investigación dedicada básicamente a las necesidades de los investigadores del Cnupc.

Esta orientación implica para la biblioteca cuestionarse continuamente la evolución de las necesidades tanto de los investigadores como las de los gestores de la investigación producida en el campus. Aparentemente, las necesidades del investigador como consumidor de información están claramente definidas (Borrego, 2015), pero para ayudarle en todas las facetas de su trabajo, es preciso mantener contacto con él, visitar su despacho y dejar que nos explique su día a día. Y, desde hace ya tiempo, en nuestras entrevistas con investigadores enseguida se pone de manifiesto que son también autores.

Buena parte de su actividad se concreta en la publicación de artículos, actas, informes o libros, y, en consecuencia, muchas de las dudas que plantean giran alrededor del proceso de publicación. Una de sus principales preocupaciones es armonizar productividad, impacto, visibilidad, presupuesto y requisitos de agencias financiadoras: ¿dónde publico: revista o congreso?, ¿qué revistas y qué congresos son los más citados?, ¿puedo publicar en acceso abierto?, ¿en qué condiciones?, ¿cuál es el coste de publicación?...

Una vez completada la publicación, los investigadores introducen su referencia en DRAC (Descriptor de la recerca $i$ l'activitat acadèmica), el CRIS (current research information system) de la UPC, adjuntando además el archivo para su publicación en el depósito institucional. 
https://drac.upc.edu/info https://en.wikipedia.org/ wiki/Current_research_information_system

A partir de ahí se requiere del sistema una gestión automatizada de su currículo en las condiciones adecuadas para poder concurrir a programas de acreditación, convocatorias de sexenios, o de financiación de proyectos. Aunque el sistema permite importantes funciones -por ejemplo traspasar CVs al formato CVN (Curriculum vitae normalizado)-, la información retrospectiva es a menudo incompleta o inexistente. Por ello es frecuente que investigadores senior no dispongan de un currículo completo para uso propio o de la universidad.

Pero eso no es todo. Las unidades que realizan, gestionan o difunden la investigación necesitan disponer de datos sobre el impacto y la productividad de las publicaciones como indicador de la actividad investigadora. Aunque los rankings de universidades acostumbran a proporcionar titulares impactantes, no suelen aportar información reveladora sobre el posicionamiento en las áreas científicas concretas o sobre dinámicas de colaboración entre instituciones, que contribuya a analizar en detalle y a poner en contexto la evolución de la investigación en el campus.

Futur tiene al investigador, y no a la investigación, como pieza principal de exposición

Por todo ello, desde la BRGF se ha considerado que la publicación, su visibilidad, y el análisis de indicadores significativos de la evolución e impacto de la publicación, son los tres elementos a definir como nuevos focos de servicio directo para los investigadores del Cnupc y de la UPC en general. A continuación se describen algunas de las prestaciones que la biblioteca ofrece para afrontar con mayor eficiencia el entorno actual en el que se desarrolla la investigación en el campus.

\section{Futur, nuevo escaparate de los investigadores de la UPC}

Hablar de Futur (Cacho et al., 2013; Rovira-Fernández, 2014), el portal de la producción científica de los investiga- dores de la UPC, significa obligatoriamente citar DRAC, un CRIS evolucionado e interoperable que se nutre de bases de datos de la propia universidad -entre ellas UPCommons, el depósito institucional de la UPC-, de recursos externos y de la información introducida por los propios investigadores (Prieto-Jiménez et al., 2010; (Cacho-Figueras; Prieto-Jiménez, 2010).

http://futur.upc.edu

$D R A C$ es la fuente de datos principal de Futur-aunque éste también recoge datos de otras unidades y servicios de la organización (Servei de Personal, Centre de Transferència de Tecnologia, Oficina de Doctorat, etc.)-. Esta alimentación garantiza coherencia y calidad gracias a la corrección y validación de los datos bibliográficos en el CRIS que corre a cargo de los bibliotecarios temáticos, quienes han pasado así a integrarse en los procesos de gestión de la investigación de la universidad, convirtiendo esta tarea en una de las más destacadas de su trabajo. Por ejemplo, para la BRGF ha significado que durante los últimos cuatro años se han validado los datos bibliográficos de más de 9.700 publicaciones firmadas por unos 600 investigadores del Cnupc.

Así pues, a partir de DRAC y con la experiencia previa del portal Fènix.Doc (Rovira-Fernández; Prats-Prat, 2005), el Servei de Biblioteques Publicacions i Arxiu de la UPC (Sbupc) ha diseñado Futur como un escaparate para investigadores, grupos de investigación y unidades estructurales. El portal permite consultar de manera abierta y atractiva los datos 
referidos a la actividad de cada investigador, así como del conjunto de los miembros de un grupo de investigación, departamento o centro docente.

Se busca que la diferencia la marque la experiencia de consulta, porque Futur tiene al investigador y no a la investigación como pieza principal de exposición. A partir de esta premisa, se incorporan gráficas que describen su actividad investigadora según tipos, y su evolución a lo largo de los últimos años. Los enlaces a la publicación original o a la versión en acceso abierto en UPCommons, enriquecen la visita al portal. Además, la integración de APIs de Scopus y Altmetrics.com aporta datos sobre el impacto de las publicaciones. Los enlaces a redes sociales ofrecen la posibilidad de difundir a través de estos medios las actividades del investigador y de la UPC en general.

El investigador y el coordinador del grupo de investigación pueden personalizar el perfil personal o del grupo, con una fotografía o logo, y con enlaces a información adicional, como los identificadores de autor o el url de la web del grupo de investigación.

En consecuencia, al cabo de un año de su estreno, el portal se está posicionando entre los investigadores como un eficiente escaparate de su labor, que permite en muchos casos simplificar o eliminar canales o aplicaciones creados por los departamentos, en los que se venían difundiendo sus publicaciones y actividades -en los últimos meses muchos departamentos han enlazado a Futur desde sus páginas web, y el directorio de personal de la UPC incluye un enlace en el registro de los investigadores-.

http://directori.upc.edu/directori/dadesPersona.jsp?id=1000013

El éxito de Futur ha dejado también al descubierto errores y carencias en las referencias más antiguas, recogidas en su día a través de Fènix (el antiguo CRIS de la UPC). Por este motivo, bastantes investigadores, especialmente los de carrera más dilatada, han reparado en la conveniencia de actualizar y corregir los datos bibliográficos de sus publicaciones, ahora mucho más visibles.

Una de las principales preocupaciones de los investigadores es armonizar productividad, impacto, visibilidad, presupuesto y requisitos de agencias financiadoras

La BRGF apoya a estos usuarios en la tarea de actualizar los datos de su currículo en DRAC y Futur. No sólo se trata de realizar correcciones puntuales que los investigadores han solicitado explícitamente, sino que hemos propuesto a 30 profesores de la UPC, seleccionados entre los que poseen un mayor índice $\mathrm{H}$ en el $\mathrm{Cnupc}$, la mejora de la información bibliográfica visible en sus currículos.

Se trata de completar el listado de publicaciones, introduciendo a través de DRAC aquellas que hasta el momento no se habían incorporado al sistema de información, añadir los campos que faltan en referencias incompletas y eliminar duplicidades. Asimismo se corrigen y uniformizan aspectos formales y de estilo.

Además se publican en UPCommons los archivos de las publicaciones retrospectivas a las que hasta el momento no se había podido dar acceso abierto, atendiendo siempre a las condiciones marcadas por los editores.

Teniendo en cuenta el enorme volumen de producción científica de los investigadores escogidos, las tareas son realmente costosas, pero el hecho de trabajar con algunos de los elementos clave y de mayor peso específico del campus, no sólo es interesante por el prestigio que su presencia aporta a Futur. También lo

https://caminstech.upc.edu 
es porque sus referencias están compartidas por una amplia relación de coautores de la UPC que a su vez ven mejorar los datos bibliográficos de su currículo.

\section{Impulso a los identificadores únicos}

Otro aspecto al que la BRGF está ofreciendo apoyo es la creación de identificadores de autor. La UPC al igual que muchas otras instituciones, recomienda a sus profesores e investigadores la adopción de Orcid (CSUC, 2014). Nuestra función, además de ofrecer ayuda puntual a la creación de IDs, es la de rastrear Orcid y detectar los identificadores no presentes en el sistema. Esta información se traspasa a los administradores de $D R A C$, que la introducen en la ficha personal del investigador. Cabe destacar que estas mejoras adquirirán todavía más sentido cuando Orcid sea una pieza clave para el futuro Portal de Recerca de Catalunya (RosGorné et al., 2014).

En este contexto un ejemplo interesante de colaboración con una iniciativa impulsada por otra unidad, y que tiene por objetivo mejorar la publicación y/o visibilidad de la producción científica, es el proyecto TSC-Metrics del Departament de Teoria del Senyal i Comunicacions, que se propone dar una mayor visibilidad y preeminencia a los resultados de la investigación de sus miembros. Para ello, el propio departamento creó una aplicación en la que todos sus investigadores deben registrarse, indicar su identificador Researcher$I D$ y seleccionar una serie de palabras clave que definan sus áreas temáticas de especialización. TSC-Metrics realiza una búsqueda en Web of Science (WoS) a partir del conjunto de identificadores, ofreciendo como resultado el total de publicaciones de los miembros del Departamento recogidas por esta base de datos. La aplicación calcula también el índice $H$ agregado. Este indicador resulta particularmente valorado en el campo de la ingeniería de telecomunicaciones como garantía de solidez y experiencia investigadora en la búsqueda de socios en proyectos internacionales. La colaboración de la BRGF en TSC-Metrics ha consistido en ayudar al diseño inicial, pero también se ha colaborado con los profesores en el registro a Researcher-ID y WoS, y muy especialmente en la localización de sus publicaciones en esta base de datos, así como en la posterior agregación al identificador.

La búsqueda del conjunto de las publicaciones de un autor en Scopus y WoS y su vinculación, es la parte más laboriosa de la creación de identificadores, básicamente a causa de la dificultad en la recuperación de publicaciones en las que constan formas alternativas, o no habituales del nombre del mismo. También son comunes las equivocaciones en las afiliaciones y las ausencias no justificadas de registros en bases de datos. Por ello, desde la BRGF también se gestiona, para cualquier investigador del $C$ nupc, la corrección de este tipo de errores en registros de bases de datos, reportándolos a los servicios técnicos de WoS, Scopus o a editores como IEEE. Aunque el éxito en la gestión es desigual, la respuesta de los autores es siempre positiva.

\section{Asesoría sobre publicación en acceso abierto}

El interés del colectivo de investigadores por hacer más visibles sus publicaciones se ha ido incrementando. La idea de que una mayor accesibilidad redunda en un mayor impacto y notoriedad es ya mayoritariamente aceptada. En este contexto, diversos grupos de investigación han manifestado su voluntad de abrir el acceso a sus publicaciones retrospectivas.

La BRGF, conjuntamente con los coordinadores de los grupos de investigación, está examinando los archivos hasta ahora publicados en UPCommons, y está substituyéndolos, siempre que sea posible, por archivos publicables en abierto que proporcionan los autores.

La creciente motivación de los investigadores por hacer más

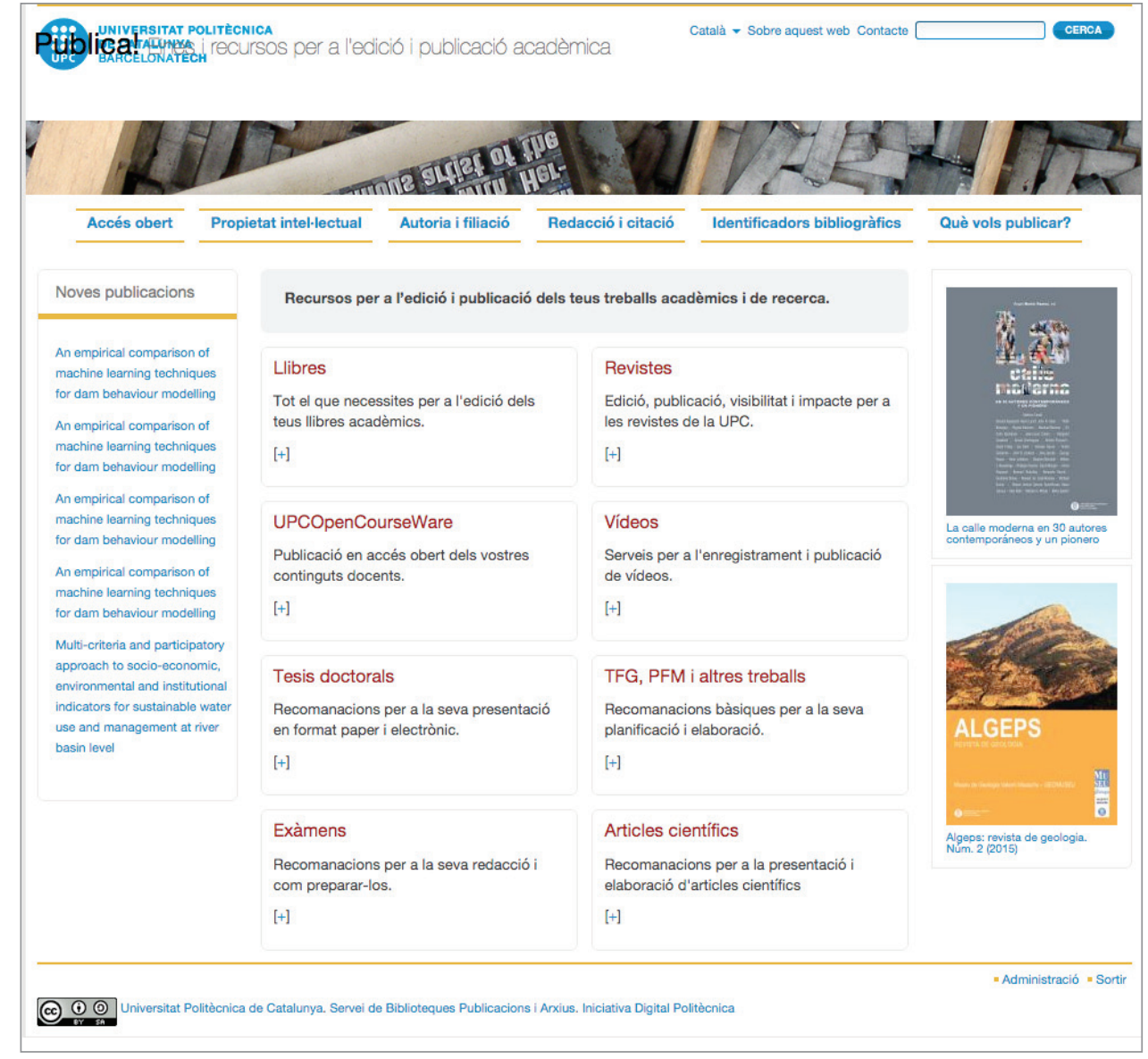

http://publica.upc.edu 
accesibles sus publicaciones, se ve fortalecida por la normativa de la UPC recomendando la publicación en acceso abierto de los resultados de la investigación (UPC, 2009; 2014a), y muy especialmente, por la obligación fijada por la Ley de la ciencia, la tecnología y la innovación (España, 2011) de publicar en acceso abierto las publicaciones fruto de proyectos financiados a cargo de convocatorias del Ministerio de Economía y Competitividad (Mineco), y del programa Horizon 2020 (H2020) de la Unión Europea para financiación de la investigación.

El requerimiento legal ha contribuido a una mayor atención por parte de los autores en las políticas de propiedad intelectual de los editores. Concretamente, hay mayor preocupación por las opciones y condiciones que los editores académicos ofrecen para publicar en acceso abierto. Los diferentes modelos de publicación están ahora en el foco de los grupos de investigación, tomando en consideración los costes de publicación y difusión en abierto por la vía dorada, la promocionada por los grandes editores académicos. Para dar respuesta a estos aspectos, ya en 2005 el Sbupc puso en marcha SEPI (Vives-Gràcia, 2005), un servicio de asesoramiento sobre temas de propiedad intelectual; y recientemente, Publica!, un portal que aglutina instrumentos y recursos útiles para la edición y publicación académica en sus diversos aspectos: propiedad intelectual, acceso abierto, condiciones de las editoriales para publicación en autoarchivo, identificadores de autor, pautas de redacción y estilo, etc.

\section{El portal se está posicionando entre los investigadores como un eficiente esca- parate de su labor}

No sólo el investigador está preocupado por los requisitos de publicación en abierto. Los gestores de proyectos nacionales y europeos de la Universidad tienen gran interés en que los investigadores conozcan y cumplan los procedimientos que marcan las agencias financiadoras. Por ello el Sbupc y el Centre de Transferència de Tecnologia trabajan conjuntamente para informar sobre las posibilidades de publicar vía UPCommons, y hacer un seguimiento de la publicación en acceso abierto por parte de los grupos beneficiarios de financiación para proyectos.

La BRGF ofrece información directa a los investigadores coordinadores de los proyectos financiados por convocatorias del Mineco y $\mathrm{H} 2020$ sobre las condiciones requeridas en cada caso -promocionando de nuevo UPCommons como repositorio para publicar gratuitamente en abierto-. La respuesta es muy positiva, y se aprecia como a partir de la información proporcionada por la Biblioteca, algunos grupos de investigación diseñan una nueva estrategia de difusión de su trabajo, tomando como base UPCommons, y optimizando así la distribución del presupuesto del proyecto.

\section{Análisis de la publicación científica en el campus}

Entre 2004 y 2006 el SBUPC publicó anualmente un estudio sobre la producción científica en los distintos ámbitos de la UPC, comparándola con la que se generaba en otras institu- ciones internacionales. Prolongando y modificando aquella idea, la BRGF empezó en 2008 a publicar estudios sobre la producción científica vinculada a los distintos centros del Campus Nord en comparación con otras universidades e instituciones de investigación de ámbito internacional.

Los objetivos principales de estos análisis son:

- conocer dónde se sitúa el centro de la UPC analizado respecto a otras entidades de referencia de su mismo ámbito temático;

- visibilizar la investigación vinculada al centro UPC analizado, y marginalmente, al resto de centros con los que se compara;

- apoyar a los órganos de gestión de la universidad, y especialmente a los de las escuelas, en la toma de decisiones vinculadas a la orientación de la investigación en sus ámbitos competenciales.

Los estudios se han venido realizando para todas las escuelas con sede en el Campus y también para los departamentos de áreas temáticas transversales a todos los campus de la UPC, como las matemáticas y la física. Lo más interesante es que metodológica y estructuralmente todos los informes tienen las mismas características básicas -que pueden incorporar variaciones en cada caso a demanda de los centros implicados-:

- se basan en Web of Science (WoS);

- se analizan la cantidad y el impacto (análisis sobre citas) de la producción científica;

- se comparan universidades de ámbito europeo y/o mundial, pero también se incluyen las que se consideran de referencia en el ámbito estatal;

- se ofrece información sobre las revistas y congresos predominantes en la publicación en el área temática que se analiza.

Es decir, se ha creado un modelo a partir del cual se pueden elaborar análisis tipo para cualquier unidad que esté interesada, incorporando ligeras variaciones cuando el centro en cuestión así lo requiera.

Todos los estudios se realizan como una coautoría entre la $B R G F$ y la unidad que encarga el estudio. La Biblioteca ofrece y aplica sus conocimientos técnicos y elabora el documento, pero la base sobre la que se trabaja -las subject categories de WoS y las universidades que se comparan- se deciden conjuntamente entre la unidad impulsora del estudio y la Biblioteca. Esto permite aprovechar un conocimiento de estos elementos que la $B R G F$ no posee con tanta profundidad y adaptar el trabajo a aquello que realmente es de interés para los principales destinatarios de los análisis efectuados.

A raíz del interés suscitado por estos estudios sectoriales, el equipo rectoral de la UPC encarga puntualmente a la $B R G F$ análisis concretos relacionados con aspectos sobre los que necesita información para la toma de decisiones en procesos vinculados a las relaciones externas y la internacionalización de la universidad.

Se trata, por ejemplo, de detectar cuáles han sido las coautorías entre los investigadores UPC y los de otros ámbitos geográficos donde hay universidades con las cuales la UPC está negociando algún tipo de colaboración, o de analizar la 
fortaleza de determinados ámbitos temáticos de alguna(s) universidad(es) con la que se prevé llegar a acuerdos, etc.

En estos casos el análisis se realiza sobre la base de datos que la biblioteca considera más efectiva para el caso y no hay una estructura preestablecida. Ésta se adapta a los intereses del demandante en ese momento preciso y varía en función de los objetivos que se pretendan conseguir en la relación con las instituciones implicadas.

No sólo el investigador está preocupado por los requisitos de publicación en abierto

\section{Conclusiones}

La actividad de las bibliotecas universitarias tiene que estar determinada por los objetivos y estrategias de las instituciones de las que forman parte. En un contexto socioeconómico y normativo cada vez más exigente, es necesario interrogarse sobre qué valores se aportan a la institución para potenciar su actividad investigadora.

La respuesta pasa por mantener una constante atención en las necesidades de los miembros de la comunidad, y por la observación de los procesos de la investigación y del espacio que ocupan unidades y servicios en su gestión. Teniendo en cuenta la multiplicidad de entornos en los que se lleva a cabo la investigación académica, es cada vez más importante mantener el servicio a la investigación pero concentrándose en el apoyo al investigador. La personalización de la oferta y el continuo seguimiento de cómo se desarrolla el servicio concreto, para abarcar el máximo de ámbitos en los procesos de la publicación científica, se muestran como claves del éxito en las actividades de la biblioteca de una universidad con vocación investigadora.

Por otra parte, en la compleja estructura de los centros universitarios, la transversalidad -característica de los servicios de biblioteca- es un factor en alza en el servicio a la investigación. La proactividad y la agilidad en establecer lazos con diferentes agentes es la clave para dotar de sentido y eficacia a nuestros servicios. La colaboración interbibliotecaria dentro y fuera de la organización que acoge una determinada biblioteca- es importante, pero también lo es el trabajo conjunto con otros actores de la universidad para conseguir los servicios de calidad a los que la biblioteca universitaria debe aspirar.

Teniendo en cuenta estas premisas, la BRGF ha implementado una suite de servicios que tienen como objetivo básico poner a la biblioteca en los circuitos propios de la actividad de los investigadores, aportando valor mediante la captación, revisión, tratamiento, preservación y difusión de la producción científica en su campus; así como asesorando y ayudando a los investigadores en el curso cambiante de la comunicación científica.

Algunos contextos que se vislumbran en los próximos años parecen anunciar que el apoyo a la investigación vendrá marcado por tres factores que seguirán poniendo al investi- gador-autor en el eje de nuestro trabajo:

- aplicación de estrategias en la colaboración científica internacional, cuyos resultados se deberán hacer evidentes a través de indicadores de impacto y posición (tradicionales y alternativos);

- emergencia de nuevas exigencias de transparencia que se plasman ya en la demanda de gestionar, publicar y preservar de los datos de investigación (González et al., 2013);

- evolución de los modelos de negocio de la comunicación científica, adaptados por completo a un entorno abierto y social.

\section{Bibliografía}

Birnholtz, Jeremy P. (2007). "When do researchers collaborate? Toward a model of collaboration propensity". Journal of the American Society for Information Science and Technology, v. 58, n. 14, pp. 2226-2239.

http://dx.doi.org/10.1002/asi.20684

Borrego, Ángel (2015). Comportament informatiu del professorat de les universitats catalanes: dades de la Universitat Politècnica de Catalunya (UPC). Barcelona: Consorci de Serveis Universitaris de Catalunya.

http://hdl.handle.net/2117/26482

Cacho-Figueras, Yolanda; Clavero-Campos, Javier; PrietoJiménez, Antonio-Juan; Martínez-Trujillo, Dídac; RoviraFernández, Anna; Serrano-Muñoz, Jordi (2013). "Futur: el nou portal de la producción científica de la Universitat Politècnica de Catalunya". Ítem, n. 57, pp. 146-156.

http://www.raco.cat/index.php/Item/article/viewFile/269709/358083

Cacho-Figueras, Yolanda; Prieto-Jiménez, Antonio-Juan (2010). "La publicació científica de la UPC: integració de sistemes d'informació, política institucional d'accés obert i nous rols per a la biblioteca". En: $12^{\text {es }}$ Jornades catalanes d'informació i documentació, 2010, Barcelona, pp. 1-18. http://upcommons.upc.edu/e-prints/handle/2117/9733

CSUC (2014). Universidades catalanas acuerdan el uso del identificador orcid para sus investigadores, nota de prensa. Consorci de Serveis Universitaris de Catalunya.

http://www.csuc.cat/sites/default/files/docs/np06_orcid_es.pdf

España (2011). “Ley 14/2011, de 1 de junio, de la ciencia, la tecnología y la innovación". BOE, n. 131, pp. 54387-54455. http://www.boe.es/boe/dias/2011/06/02/pdfs/BOE-A-2011-9617. $p d f$

Fecyt (2014). Indicadores bibliométricos de la actividad científica española 2011. Madrid: Fundación Española para la Ciencia y la Tecnología.

http://www.fecyt.es/es/node/2561/pdf-viewer

González, Luis-Millán; Saorín, Tomás; Ferrer-Sapena, Antonia; Aleixandre-Benavent, Rafael; Peset, Fernanda (2013). "Gestión de datos de investigación: Infraestructuras para su difusión". El profesional de la información, v. 22, n. 5, pp. 415-423. http://dx.doi.org/10.3145/epi.2013.sep.06

INE (2015). Estadística sobre actividades en I+D. Resultados definitivos. Año 2013. Madrid: Instituto Nacional de Estadística. http://www.ine.es/prensa/np889.pdf 
Iribarren-Maestro, Isabel; Grandal, Teresa; Alecha, María; Nieva, Ana; San-Julián, Teresa (2015). “Apoyando la investigación: nuevos roles en el servicio de bibliotecas de la Universidad de Navarra". El profesional de la información, v. 24, n. 2, pp. 131-137.

http://dx.doi.org/10.3145/epi.2015.mar.06

Michavila, Francisco (dir.) (2013). La Universidad española en cifras 2012. Madrid: Conferencia de Rectores de las Universidades Españolas (CRUE).

http://www.crue.org/Publicaciones/Documents/UEC/LA_ UNIVERSIDAD_ESPANOLA_EN_CIFRAS.pdf

Nández, Gemma; Borrego, Ángel (2013). "Use of social networks for academic purposes: a case study". The electronic library, v. 31, n. 6, pp. 781-791.

http://dx.doi.org/10.1108/EL-03-2012-0031

Prieto-Jiménez, Antonio-Juan; Cacho-Figueras, Yolanda; Ínigo-Robles, Ruth; Rovira-Fernández, Anna; Serrano-Muñoz, Jordi (2010). "Integrating research output in UPC repositories". En: 5a Open repositories Madrid, pp. 1-19. http://hdl.handle.net/2117/8389

Ros-Gorné, Ramon; Anglada, Lluís; Reoyo, Sandra; De-laVega, Ricard (2014). "Let's do data research work: the creation of a portal with research information from Catalan Universities". En: Open repositories, Helsinki. DSpace Interest Group presentations, pp. 1-26.

http://hdl.handle.net/11366/333

Rovira-Fernández, Anna (2014). "Futur, el portal de la producció científica dels investigadors de la UPC Barcelona". En: $3^{\text {es }}$ Jornades sobre Gestió de la Informació Científica, Barcelona, pp. 1-17.

http://blogs.iec.cat/observatori/wp-content/uploads/ sites/2/2014/04/Anna-Rovira.pdf

Rovira-Fernández, Anna; Prats-Prat, Jordi (2005). "Fènix-
Doc: accés a la producció científica de la UPC", ppt. En: $1^{\text {er }}$ Espai CBUC Barcelona, pp. 1-15.

http://hdl.handle.net/2117/1596

UPC (2008). De les relacions internacionals a la internacionalització: Pla de política internacional 2008-2015. Universitat Politécnica de Catalunya, Vicerrectorat de Política Internacional.

https://www.upc.edu/sri/estrategia/ppi_RETALLAT_catala. $p d f$

UPC (2009). Acord núm. 171/2009 del Consell de Govern pel qual es ratifica la Política Institucional d'Accés Obert: Accés, impacte i preservació de la producció acadèmica de la UPC a internet. Universitat Politècnica de Catalunya, Vicerrectorat de Recerca i innovació.

http://publica.upc.edu/sites/default/files/arxius_site/file/ open_acces/mandatcat.pdf

UPC (2014a). Acord núm. 177/2014 del Consell de Govern pel qual s'aprova l'assignació de punts PAR únicament per publicacions en accés obert. Universitat Politécnica de Catalunya, Vicerrectorat de Política de Recerca.

$h t t p: / / g o o . g l / j 8 p 4 R w$

UPC (2014b). Informe d'indicadors de l'activitat de recerca PAR i PATT 2013. Universitat Politécnica de Catalunya, Vicerrectorat de Política de Recerca.

http://goo.gl/nKafL8

Van-Noorden, Richard (2014). “Online collaboration: scientists and the social network". Nature, August, v. 512, pp. 126-129.

http://dx.doi.org/10.1038/512126a

Vives-Gràcia, Josep (2005). "El nuevo Servicio de Propiedad Intelectual en las bibliotecas de la UPC". En: 5o Workshop Rebiun sobre proyectos digitales, Barcelona, pp. 1-15 http://hdl.handle.net/2117/1651

\section{Da visibilidad a tu trabajo depositándolo en e-LIS, el mayor repositorio internacional sobre biblioteconomía, documentación y comunicación}

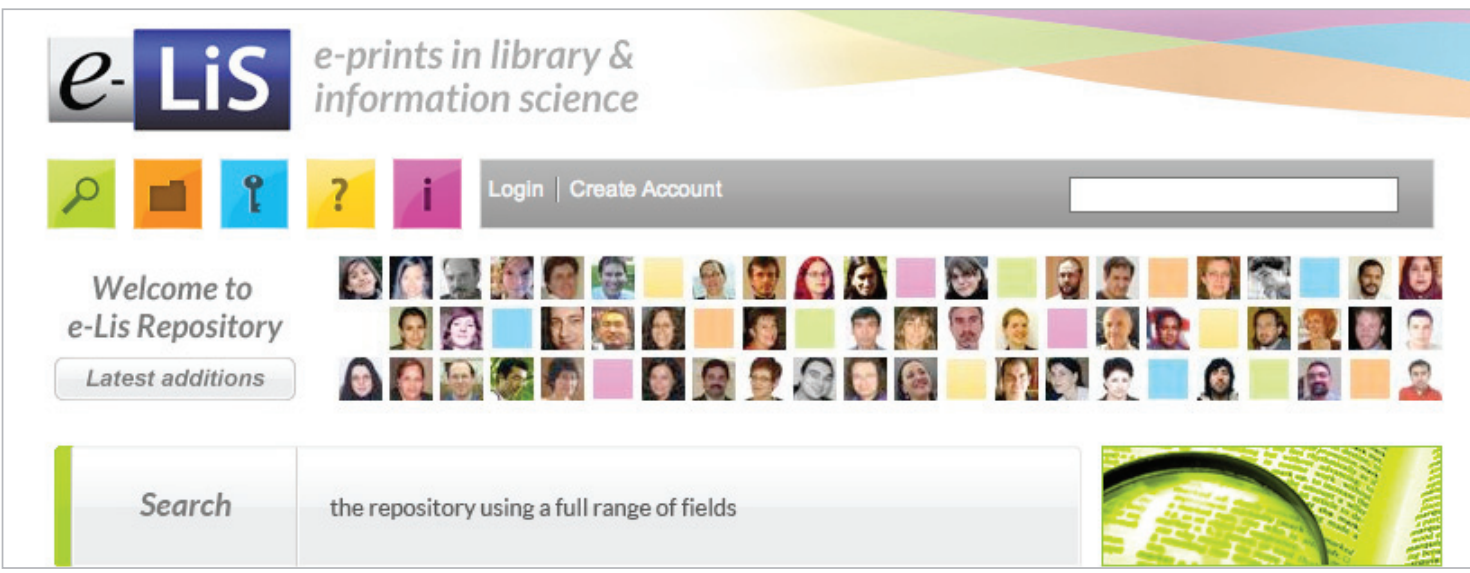

http://eprints.rclis.org 